\title{
Phase Entrainment of Human Delta Oscillations Can Mediate the Effects of Expectation on Reaction Speed
}

\author{
Gábor Stefanics, ${ }^{1,2,3 *}$ Balázs Hangya, ${ }^{4 *}$ István Hernádi, ${ }^{2}$ István Winkler, ${ }^{1,5}$ Péter Lakatos, ${ }^{1,6}$ and István Ulbert ${ }^{1,7,8}$ \\ ${ }^{1}$ Institute for Psychology, Hungarian Academy of Sciences, H-1068, Budapest, Hungary, ${ }^{2}$ Department of Experimental Zoology and Neurobiology, \\ University of Pécs, H-7624, Pécs, Hungary, ${ }^{3}$ Centre for Neuroscience in Education, University of Cambridge, Cambridge CB2 8PQ,United Kingdom, \\ ${ }^{4}$ Department of Cellular and Network Neurobiology, Institute of Experimental Medicine, Hungarian Academy of Sciences, H-1083, Budapest, Hungary, \\ ${ }^{5}$ Institute of Psychology, University of Szeged, H-6722, Szeged, Hungary, ${ }^{6}$ Cognitive Neuroscience and Schizophrenia Program, Nathan Kline Institute, \\ Orangeburg, New York 10962, ${ }^{7}$ Faculty of Information Technology, Péter Pázmány Catholic University, H-1083, Budapest, Hungary, and ${ }^{8}$ National Institute \\ of Neuroscience, H-1145, Budapest, Hungary
}

The more we anticipate a response to a predictable stimulus, the faster we react. This empirical observation has been confirmed and quantified by many investigators suggesting that the processing of behaviorally relevant stimuli is facilitated by probabilitybased confidence of anticipation. However, the exact neural mechanisms underlying this phenomenon are largely unknown. Here we show that performance changes related to different levels of expectancy originate in dynamic modulation of delta oscillation phase. Our results obtained in rhythmic auditory target detection tasks indicated significant entrainment of the EEG delta rhythm to the onset of the target tones with increasing phase synchronization at higher levels of predictability. Reaction times correlated with the phase of the delta band oscillation at target onset. The fastest reactions occurred during the delta phase that most commonly coincided with the target event in the high expectancy conditions. These results suggest that low-frequency oscillations play a functional role in human anticipatory mechanisms, presumably by modulating synchronized rhythmic fluctuations in the excitability of large neuronal populations and by facilitating efficient task-related neuronal communication among brain areas responsible for sensory processing and response execution.

\section{Introduction}

Oscillatory brain activity represents rhythmic changes in baseline excitability of neuronal populations at a variety of temporal and spatial scales (Başar, 1980; Buzsáki and Draguhn, 2004; Buzsáki, 2006) affecting perception and action. Very slow fluctuations $(\sim 0.1 \mathrm{~Hz})$ in EEG activity, cyclically modulating cortical gross excitability (Vanhatalo et al., 2004) have been shown to predict stimulus detection performance and amplitudes of $1-40 \mathrm{~Hz}$ oscillations (Monto et al., 2008). Amplitudes of prestimulus medial temporal theta oscillations in a memory task were higher for later-recalled than for later-forgotten words (Guderian et al., 2009). Momentary alpha amplitude (Romei et al., 2008; van Dijk et al., 2008) and phase (Busch et al., 2009; Mathewson et al., 2009) in visual areas were found to predict visual perceptual performance. In the human motor system, the phase of beta oscillations was observed to modulate input gain (van Elswijk et al., 2010). The large variability in evoked cortical responses to the same

\footnotetext{
Received Feb. 8, 2010; revised May 28, 2010; accepted July 6, 2010.

This research was supported by the Hungarian Scientific Research Fund (Grant OTKA K-81357 to I.U.) and the National Institutes of Health (Grant R21 DC010415 from the National Institute on Deafness and Other Communication Disorders to P.L.). We thank George Karmos and Viktor Varga for critical discussions and invaluable comments on the manuscript.

*G.S. and B.H. contributed equally to this work.

Correspondence should be addressed to Gábor Stefanics, Institute for Psychology, Hungarian Academy of Sciences, 83-85 Szondi Street, H-1068, Budapest, Hungary. E-mail: gstefan@cogpsyphy.hu.

DOI:10.1523/JNEUROSCI.0703-10.2010

Copyright $\odot 2010$ the authors $\quad 0270-6474 / 10 / 3013578-08 \$ 15.00 / 0$
}

stimulus can be explained by the momentary state of ongoing neuronal activity (Arieli et al., 1996; Kruglikov and Schiff, 2003; Lakatos et al., 2005; Haslinger et al., 2006; Womelsdorf and Fries, 2006; Haider et al., 2007; Hasenstaub et al., 2007). Phase entrainment of rhythmic electric brain activity to periodic stimulation occurs spontaneously (Adrian and Matthews, 1934; Walter et al., 1946; Galambos et al., 1981; Will and Berg, 2007). Additionally, the entrainment of cortical delta-band $(0.5-3 \mathrm{~Hz})$ oscillations has been recently described and suggested as a key mechanism of selective attention in macaque monkeys (Lakatos et al., 2008). In their study, Lakatos et al. showed that the phase locking of lowfrequency cortical oscillations to attended rhythmic auditory or visual stimulus streams can result in elevated response gain and faster reaction times (RT).

Attention and expectation are two complementary mechanisms of processing large amounts of stimulus information using limited capacities (Summerfield and Egner, 2009). They both lead to more efficient detection and recognition of behaviorally relevant stimuli. Expectation influences the processing of stimuli on the basis of prior likelihood, whereas attention modifies stimulus processing on the basis of motivational relevance. "Topdown" attentional influences are directed toward task-relevant features and often interact with temporal expectations (Nobre et al., 2007). One possibility is that temporal predictability allows the brain to optimize the timing of the processing of the expected stimulus and thus make them faster and more efficient (Bendixen et al., 2009). It has recently been proposed (Schroeder and Lakatos, 
A

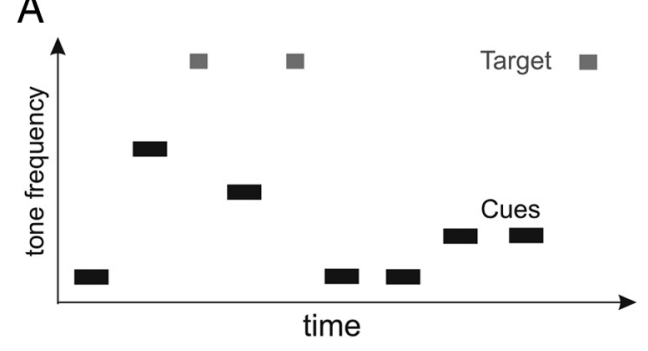

C

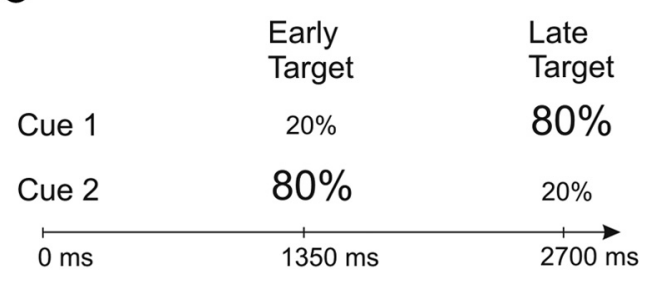

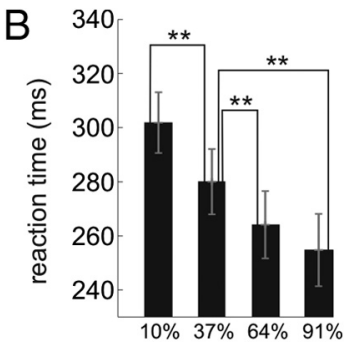

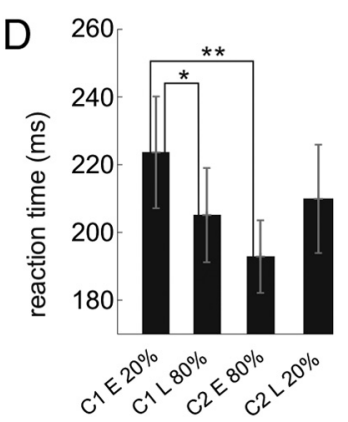

Figure 1. Reaction time correlates with the level of expectancy. $\boldsymbol{A}$, Auditory target detection paradigm of experiment 1. Four different cue tones (black) predicted the target tone (gray) to be the next stimulus with increasing probability paralleling the increase of cue-tone frequencies ( $p=0.1, p=0.37, p=0.64$ and $p=0.91$ ). Participants were to press a response key to the target tone. $\boldsymbol{B}$, Reaction times (RT) significantly decreased with increasing predictability of the target $\left(n=13 ;\right.$ ANOVA, $^{2}<10^{-9}$, with Tukey's post hoc tests, $p<0.01$ ). Error bars indicate SEM. C, In experiment 2, two different cue tones predicted the timing of the target tone with different probabilities. An early target ( $1350 \mathrm{~ms}$ after the cue) was delivered with $p=0.2$ after cue 1 and with $p=0.8$ after cue 2 . 0 n trials with no early target, a late target ( $2700 \mathrm{~ms}$ after the cue) was delivered ( $p=0.8$ after cue 1 and $p=$ 0.2 after cue 2). $\boldsymbol{D}$, The early-target RT was significantly shorter after cue 2 ( $p=0.8$ probability, third bar) than after cue 1 ( $p=$ 0.2 probability, first bar) ( $p<0.01$, Tukey's post hoc test). In the absence of an early target, delivery of the late target could be predicted with $100 \%$ certainty, resulting in faster RTs compared with the early target with $p=0.2$ (following cue $1, p<0.05$ and cue $2, p=0.08)$. C1, cue $1 ; C 2$, cue2; $\mathrm{E}$, early target; L, late target. ${ }^{*} p<0.05 ;{ }^{* *} p<0.01$.

2009) that in various ecologically valid stimulus configurations, such as biological motion and vocal communication (reviewed by Schroeder et al., 2008), when the attended stimuli have a rhythmic or predictable temporal structure, the brain operates in a "rhythmic mode," in which low-frequency rhythms provide a general oscillatory framework for sensory processing. However, the brain mechanisms underlying predictive processing are still poorly understood.

Here we tested the hypothesis that oscillatory neural activity may mediate the effects of expectation on the processing of taskrelevant stimuli. We applied an auditory target detection paradigm, in which four cue tones of different frequencies predicted the probability of the next stimulus to be the target event while EEG was recorded. To investigate the effect of expectancy on delta activity, we detected the phase of ongoing delta oscillation at target stimulus onset. Delta rhythm was found to significantly entrain to target onset preceding stimulus processing, with increasing "accuracy" at higher expectancy levels. Our results provide the first evidence that the entrainment of cortical delta oscillations to a predicted stimulus is a key determinant of response gain in healthy humans. Furthermore, we show that the strength of phase-entrainment varies systematically with the probability of the target event, thus, it is not only a mechanistic consequence of the rhythmic structure within the stimulus stream, but it may depend on the behavioral significance of the expected events.

\section{Materials and Methods}

Participants. Two auditory target detection tasks were performed by two groups of 13 and 11 healthy human volunteers (for age and gender composition, see supplemental Table 1, available at www.jneurosci.org as supplemental material). None of the subjects had any history of neurological or hearing disorders. The protocol of the study was approved by the Ethical Committee of the University of Pécs. The recordings were performed at the Electrophysiological Laboratory of the Department of Experimental Zoology and Neurobiology, University of Pécs, Hungary. All subjects gave their written informed consent after the nature of the experiment had been fully explained.

Stimulation. The auditory stimuli were presented via headphones at $70 \mathrm{~dB}$ sound pressure level while EEG was recorded. In the first experiment, tones were delivered with random stimulus onset asynchronies (SOAs) varying between 1200 and $1500 \mathrm{~ms}$ at a mean repetition rate of $1350 \mathrm{~ms}$. Four cue tones of different frequencies $(1046,1174,1318$, and $1480 \mathrm{~Hz}$; $150 \mathrm{~ms}$ duration; $10 \mathrm{~ms}$ rise/fall times) predicted the probability $(10 \%, 37 \%, 64 \%, 91 \%)$ of the next stimulus to be the target tone (1975 $\mathrm{Hz}$ frequency; $50 \mathrm{~ms}$ duration; $10 \mathrm{~ms}$ rise/fall times) (Fig. 1A). The cue tones' frequencies were chosen to be separated by one semitone, whereas the target tone was two semitones higher than the highest cue tone, which enabled the subject to carry out the task easily. In our second experiment, two cue tones of distinct frequencies (1046 and $1318 \mathrm{~Hz} ; 150 \mathrm{~ms}$ duration; $10 \mathrm{~ms}$ rise/fall times) were used to predict the probability ( $20 \%$ and $80 \%$ ) of the target stimulus $(1975 \mathrm{~Hz} ; 50 \mathrm{~ms}$ duration; 10 $\mathrm{ms}$ rise/fall times) to appear at either of two fixed time points (early target at $1350 \mathrm{~ms}$ and late target at $2700 \mathrm{~ms}$ ) (see Fig. 1C; supplemental Fig. 1, available at www.jneurosci.org as supplemental material). In both experiments, the participants were informed about the meaning of the cues and they were instructed to quickly depress a button for target tones. A 5-min training block was applied before the experiments to allow subjects to learn the task. A total of 400 responses to targets ( 100 for each cue condition) were collected from each subject in the first and 1000 in the second experiment (500 for each cue condition).

Recording. EEG was recorded from F3, Fz, F4, C3, Cz, C4, P3, Pz, P4, T3, T4, $\mathrm{O} 1$ and $\mathrm{O}_{2}$ sites (according to the international 10-20 system) and two bipolar EOG electrodes placed above and below the left and right outer canthi, respectively. EEG was referred to the nose, an electrode placed on the forehead served as the ground. Impedances were kept below $5 \mathrm{k} \Omega$. Recording was continuous with an analog bandpass from $0.16 \mathrm{~Hz}$ to $150 \mathrm{~Hz}$ at a sampling rate of $1 \mathrm{kHz}$ in the first and $0.5 \mathrm{kHz}$ in the second experiment. We report the $\mathrm{Fz}, \mathrm{Cz}$, and Pz electrodes throughout the paper. Data from $\mathrm{Cz}$ was selected for creating the plots as it is the most representative electrode for our findings. Cz and frontocentral electrodes in general are the most favorable scalp locations for auditory experiments as sources in the auditory cortex project onto this area. We used the left central (C3) and right central (C4) electrodes for testing potential lateralization of the delta phase effect.

Behavioral data analysis. Participants performed both tasks easily. ANOVA was calculated from behavioral data. In experiment 1 , the oneway ANOVA (condition [ $10 \%$ vs $34 \%$ vs $64 \%$ vs $91 \%$ ]) revealed a significant effect $\left(F_{(3,36)}=32.819, p<10^{-9}\right)$ of expectancy on reaction time, which was explained by a gradient of significant differences between $10 \%<34 \%<64 \%$ (Tukey's test). Figure $1 B$ shows the observed significant differences in reaction time between conditions. Subjects responded to $99.76 \%$ of the targets in experiment 1 , the ANOVA of the number of missed trials (condition [ $10 \%$ vs $34 \%$ vs $64 \%$ vs $91 \%]$ ) revealed no significant differences between conditions $(p=0.36)$. ANOVA of reaction times in experiment 2 (one-way ANOVA (condition [cue 1 - early target vs cue 1 -late target vs cue 2 - early target vs cue 
2-late target]) revealed a significant effect $\left(F_{(3,30)}=5.963, p<0.01\right)$ showing that responses were faster when expectancy was higher. A post hoc Tukey's test revealed that the effect was caused by significantly higher reaction times for the less expected targets in the first position following cue 1 (cue 1-early target condition) compared with the more expected target in the second position following cue 1 (cue 1 -late target condition, $p<0.05$ ) and the target in the first position following cue $2(p<0.01)$. Comparison with the cue 2 -late target showed a marginal $p$ value of 0.08 (Fig. $1 D$ ). It should be noted, however, that due to perfect contingency of the target tone to both cues, in those trials when an early target was not delivered, participants dynamically updated their subjective expectancy for the late targets (100\%), which explains the similarity of response speed for late targets to the early targets predicted with high confidence $(80 \%)$. Participants responded to $98.78 \%$ of the targets in experiment 2, the ANOVA on the proportion of missed trials yielded no significant effects.

EEG data analysis. All data were analyzed off-line on a PC using built-in and selfdeveloped functions as well as the freeware EEGLAB toolbox (Delorme and Makeig, 2004) in the Matlab (MathWorks) development environment. Epochs extending from $-1500 \mathrm{~ms}$ before to $1500 \mathrm{~ms}$ after the time-locking event were created in the first experiment and -500 $\mathrm{ms}$ before to $4050 \mathrm{~ms}$ after the time-locking event in the second. Trials with a potential change below $0.1 \mu \mathrm{V}$ or voltage exceeding \pm 75 $\mu \mathrm{V}$ were marked for rejection from further analysis. Additionally, data were visually inspected and trials containing artifacts, which survived the automatic artifact rejection procedure, were also marked for rejection.

$\mathrm{Fz}, \mathrm{Cz}$, and $\mathrm{Pz}$ electrodes were selected for analysis. We established the presence of prestimulus delta oscillations by visualizing filtered and unfiltered ERP (supplemental Figs. $2-10$, available at www.jneurosci.org as supplemental material; Fig. $2 M, N$ ) and wide-band filtered single-trial data (supplemental Figs. 12, 13 , available at www.jneurosci.org as supplemental material). Oscillatory activity in the prestimulus period was also supported by the calculation of frequency spectra (supplemental Fig. 11, available at www.jneurosci.org as supplemental material). Zero-phase shift filtering of the continuous raw EEG waveforms in the delta band $(0.5-3 \mathrm{~Hz})$ was performed using a finite impulse response filter (Matlab built-in fir1.m and filtfilt.m) and several other filters were tested to exclude the possibility of filter artifacts. We performed additional analysis and simulations to rule out the possibility that filtering artifacts significantly affected our results (see supplemental Notes, supplemental Figs. 14-16, and supplemental Tables 4-6, available at www.jneurosci.org as supplemental material). Instantaneous phase values of the filtered data were calculated using the Hilbert-transform (Gabor, 1946; Hurtado et al., 2004). Filtered and Hilbert-transformed data were epoched as described above and epochs marked as artifacts were rejected.

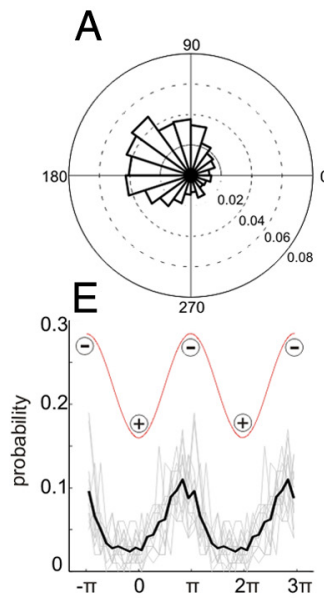

B

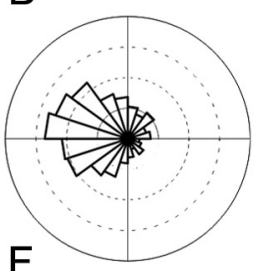

$\mathrm{F}$
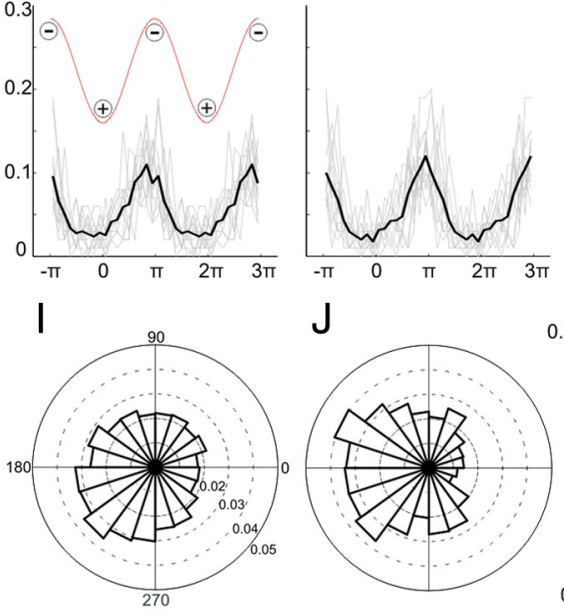

M

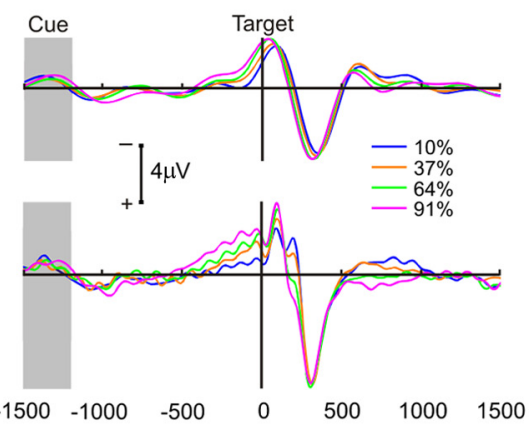

C

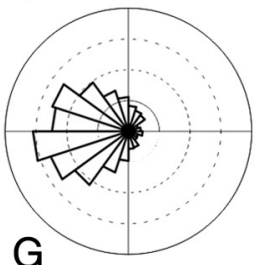

G
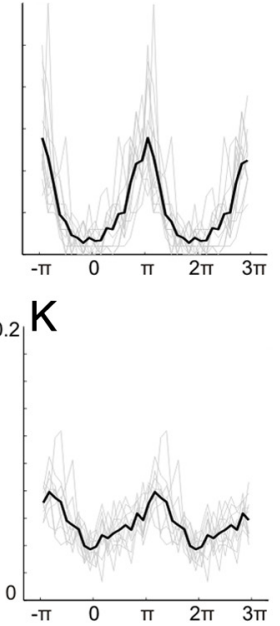

$\mathrm{D}$

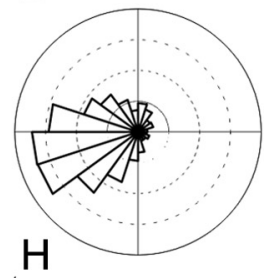

$\mathrm{H}$
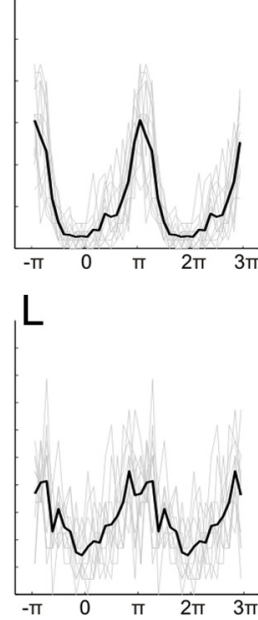

$\mathrm{N}$

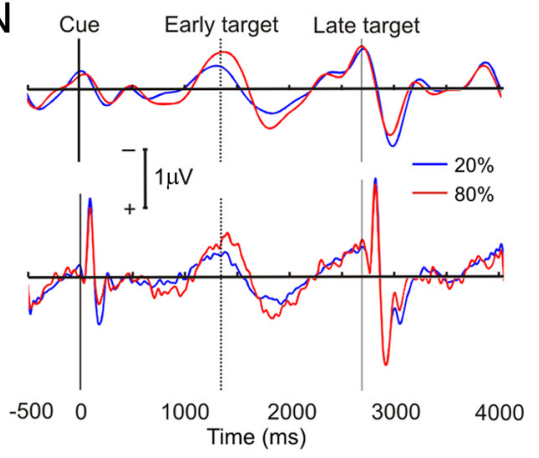

Figure 2. Phase entrainment of the cortical delta oscillation. $A-D$, Distributions of delta phase values (measured at $C z$ ) at target onset are presented on rose diagrams with the radial extent of the circle segments representing the probability of the given phase range. Trials with $10 \%(\boldsymbol{A}), 37 \%(\boldsymbol{B}), 64 \%(\boldsymbol{C})$, and $91 \%(\boldsymbol{D})$ target probabilities were separately pooled from all $(n=13)$ participants. $\mathbf{E}-\boldsymbol{H}$, Individual (gray) and average (black) phase histograms for the four different target probabilities (two cycles; idealized delta waves in red in $\mathrm{E}$, negativity is upward). Unimodal phase preference of the distributions is clearly visible, with the mean phases near the negative peak of the delta waves (from $10 \%$ to $91 \%$, in radians: $2.56,2.85,3.10,-3.03$ ). The accuracy of phase entrainment (measured as the concentration (sharpness, $\kappa$ ) of phase histograms) increased with increasing levels of target predictability ( $\kappa$ values from $10 \%$ to $91 \%: 0.74,0.82,1.07,1.19$ ). The difference between the $37 \%$ and $64 \%$ as well as between the $64 \%$ and $91 \%$ conditions was significant (permutation tests with $p$ values for the comparisons of $10 \%$ vs $37 \%, 37 \%$ vs $64 \%$ and $64 \%$ vs $91 \%: 0.094,0.0001,0.036$, respectively). I, J, Distribution of delta-phase values (measured at $(\mathrm{Z}$ ) at the time of the expected delivery of the early target in experiment 2 . Trials from late-target trials (i.e., no early target was delivered) were pooled separately for $p=0.2(\boldsymbol{I})$ and $p=0.8(\boldsymbol{J})$ early-target probability from all participants $(n=11) \cdot \boldsymbol{K}, \boldsymbol{L}$, Individual (gray) and average (black) phase histograms for $p=0.2(\boldsymbol{K})$ and $p=0.8(\boldsymbol{L})$ target probabilities. Phase values were significantly more concentrated for high- than for low-probability targets ( $p=0.024$, permutation test; $\kappa$ (mean phase): $0.27(-2.84)$ and $0.40(-3.04)$, for the $20 \%$ and $80 \%$ target probabilities, respectively). $\boldsymbol{M}$, Average EEG traces from experiment 1 aligned at target onset $(0 \mathrm{~ms})$, filtered between $0.5-3 \mathrm{~Hz}$ (top) and $0.5-20 \mathrm{~Hz}$ (bottom). The traces were averaged separately for the four different levels of target predictability (blue, 10\%; orange, 37\%; green, 64\%; purple, $91 \%$ ). Note the amplitude increase at target onset with increasing levels of target predictability. $\boldsymbol{N}$, Average filtered EEG traces from experiment 2 aligned at the presentation of the cue tone, shown separately for the two different cue tones (late-target trials, only), filtered between $0.5-3 \mathrm{~Hz}$ (top) and $0.5-20 \mathrm{~Hz}$ (bottom). The average delta-wave amplitude was markedly higher at $1350 \mathrm{~ms}$ postcue (the expected onset of the early target marked by the vertical dotted line) for cue 2 (early target by $p=0.8$; red line) than for cue 1 trials ( $p=0.2$; blue).

For statistical analysis, Rayleigh's test for uniformity of phase data (Fisher, 1993) was used to test whether phase value distributions at various time instances show any phase preference. Single-trial delta-peak amplitude and latency data were defined as the maximum amplitude and corresponding latency in the $200-480 \mathrm{~ms}$ poststimulus time window 
A

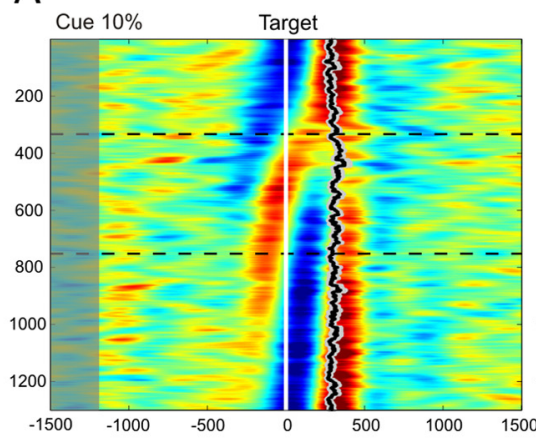

B

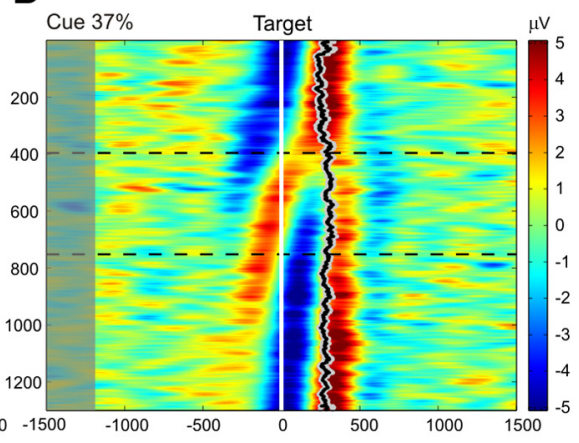

C

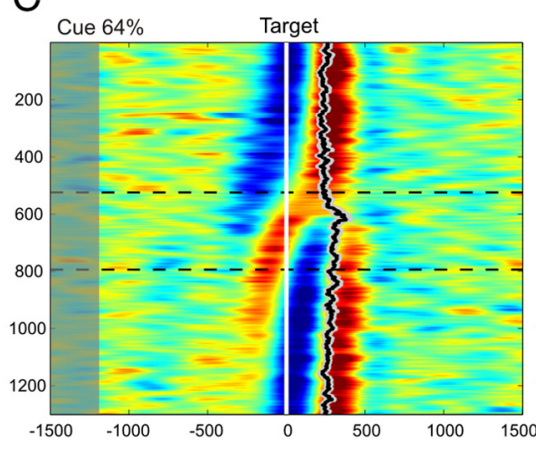

D
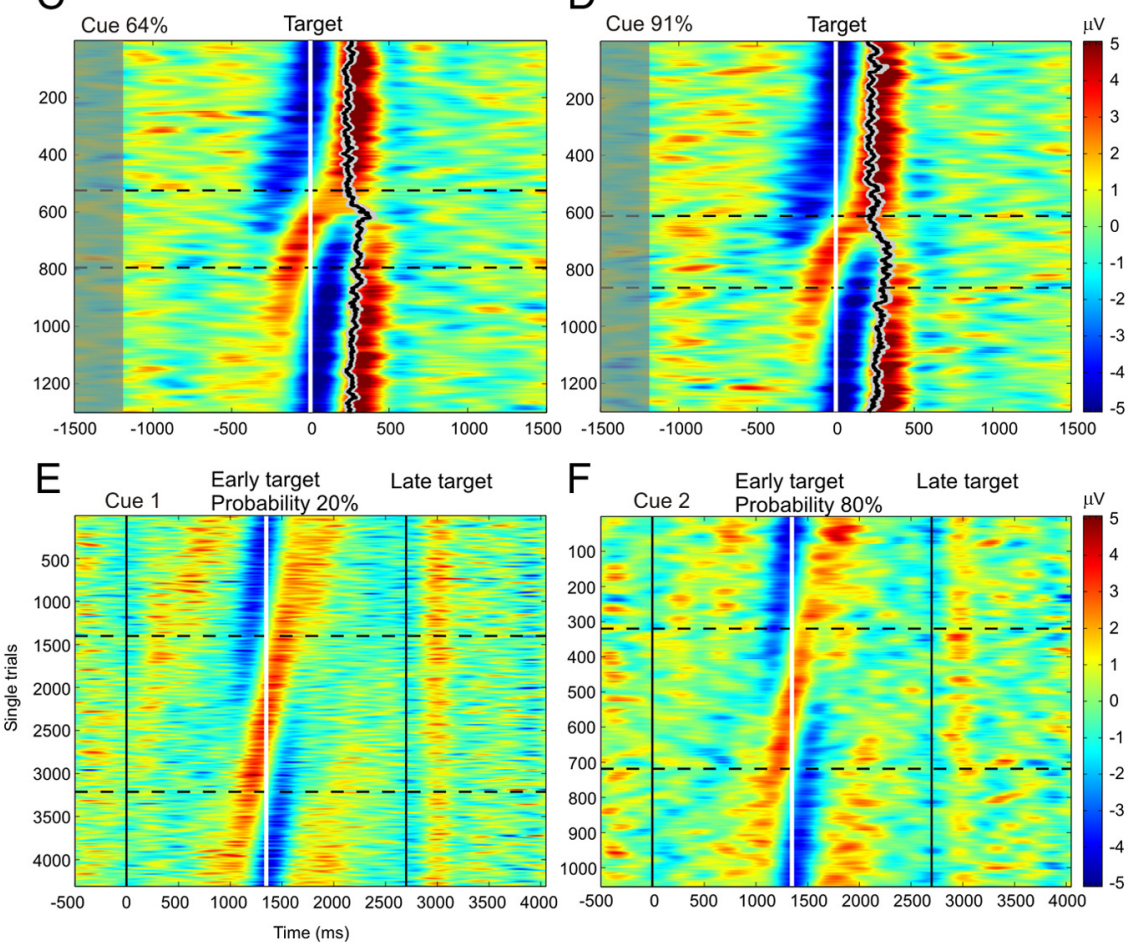

Figure 3. Phase-entrainment of delta-band EEG oscillations. $A-D$, ERP images of single-trial responses filtered between 0.5 and $3 \mathrm{~Hz}$ from experiment 1 , sorted by phase-values from $-\pi$ to $\pi$ at target onset time. Trials from all 13 subjects at $\mathrm{C} z$ site from $10 \%, 37 \%, 64 \%$ and $91 \%$ probability conditions are shown in plots $\boldsymbol{A}-\boldsymbol{D}$, respectively. $x$-axis: time in ms, $y$-axis: individual EEG traces, colors represent amplitude values. Shaded areas mark the random interval where the cues were presented between -1500 and $-1200 \mathrm{~ms}$ preceding the target. Data were smoothed using a vertical window of 20 trials. Vertical white line at $0 \mathrm{~ms}$ represent the onset of the target tone, curved vertical black lines show single reaction time values, gray lines show SEM. Area between horizontal dashed lines contains trials with positive amplitude at target onset. Note the reduction of trials falling into this region with increasing target probability and the presence of oscillatory activity during the whole epochs. $E, F$, ERP images of single-trial responses from experiment 2 from all 11 subjects, similar to plots $\boldsymbol{A}-\boldsymbol{D}$. Epochs from late-target trials, when no early target was delivered, are sorted by delta-phase values at the early $(1350 \mathrm{~ms}$ ) expected onset time (marked by vertical white lines); cue ( $0 \mathrm{~ms})$ and late target $(2700 \mathrm{~ms})$ onset times are indicated by vertical black lines. Twenty percent and $80 \%$ expectancy conditions at Position 1 are shown in $\boldsymbol{E}$ and $\boldsymbol{F}$, respectively. The proportionally smaller number of trials with less favorable phase values (between the horizontal dashed lines) in the $80 \%$ condition demonstrates that the accuracy of delta-phase entrainment increased at higher target probability.

centered on the average delta-peak measured at Cz. Linear-circular correlation (Fisher, 1993) was calculated between delta phase and reaction time, delta-peak latency and amplitude. Linear partial correlation between $X$ and $Y$ variables with a single controlling variable $Z\left(R_{X, Y \mid Z}\right)$ can be decomposed to linear correlation terms $\left(R_{X, Y}, R_{X, Z}, R_{Y, Z}\right)$ as follows:

$$
R_{X, Y \mid Z}=\frac{R_{X, Y}-R_{X, Z} R_{Y, Z}}{\sqrt{1-R_{X, Z}^{2}} \sqrt{1-R_{Y, Z}^{2}}} .
$$

Linear-circular partial correlation was performed by replacing linear correlation terms to linear-circular correlation (Fisher, 1993). Significance of phase concentration ( $\kappa$ ) differences between conditions was assessed by a permutation test with Monte Carlo randomization $(10,000$ permutations).

\section{Results}

Probability-based confidence of anticipation modulates delta phase entrainment to target stimuli

In two experiments, we measured the effects of the predictive value of cue stimuli on behavioral responses and the entrainment of delta-band oscillations to the expected occurrence of targets. In experiment 1 (Fig. $1 A$ ), four cue tones of different pitch predicted the occurrence of the target tone within the next 1200-1500 ms (average: $1350 \mathrm{~ms}$ ) with different probabilities $(p=0.1, p=0.37, p=0.64, p=$ $0.91)$. The cue was either followed by the target tone or another cue within the predicted period. Healthy human participants $(n=13)$ were required to depress a response key for targets as fast as possible. In line with previous observations (Hick, 1952; Hyman, 1953; Gehring et al., 1992; Ortiz et al., 1993; Nobre et al., 2007; van Elswijk et al., 2007), reaction times (RT) decreased with increasing target probability, indicating that participants relied on the predictive power of cue information (Fig. $1 B$ ).

Delta-band oscillations extracted from the EEG signal (see Materials and Methods) recorded at midline electrode locations $(\mathrm{Fz}, \mathrm{Cz}, \mathrm{Pz}$; figures show data from $\mathrm{Cz}$ ) preceded the target onset and also followed the ERP elicited by the target stimulus (Figs. 2M, 3A-D; supplemental Figs. $2-7,13 A-D$, available at www.jneurosci. org as supplemental material). Delta oscillation was significantly entrained to the target in all participants as was shown by the significant phase preference observed in the distribution of delta-oscillation phase values calculated at the target onset (Rayleigh's tests with $p<0.05$, supplemental Table 2, available at www. jneurosci.org as supplemental material). Strikingly, phase-locking significantly increased from 0.37 to 0.91 target probabilities (permutation tests with at least $p<$ 0.05; Fig. $2 A-H)$. Thus, the more predictable the target stimulus was, the more precisely the phase of delta oscillations was locked to the expected time of stimulus onset.

\section{Phase entrainment of delta oscillation to the time of expected target events}

Given that in experiment 1, different cues appeared with different sequential probabilities, in the second experiment, the probabilities of the cues within the sequence were set equal and we manipulated the cue-to-target temporal relationship (Fig. 1C; 

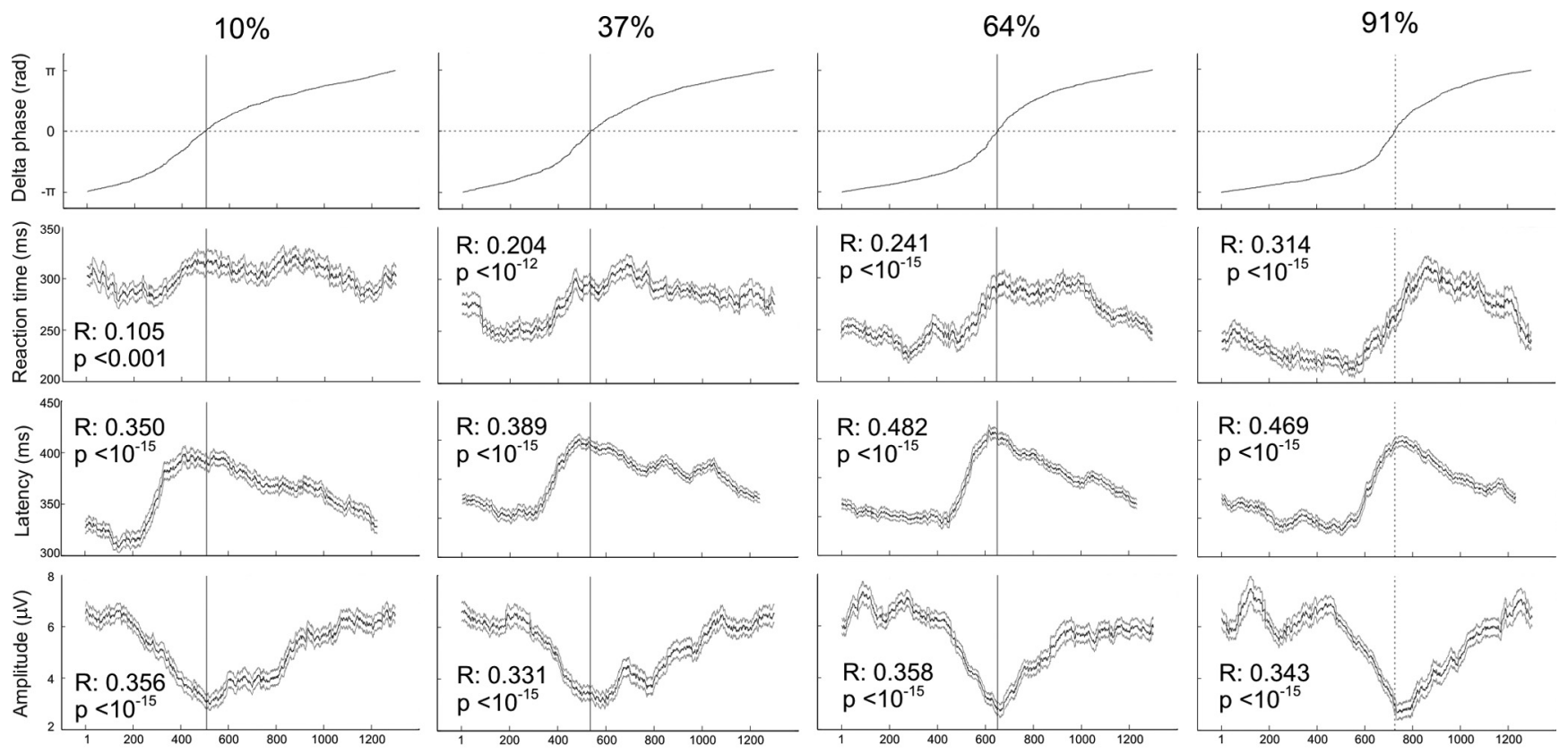

Delta phase sorted trials

Figure 4. Task performance is correlated with delta phase modulation. First row, Central (Cz) delta-phase values measured at target onset, pooled and sorted in ascending order from all trials of all participants in experiment 1 (1300 trials), separately for the four levels of target probability (from the left: 10\%, 37\%,64\%, 91\%). The nonlinearity of the curves is the consequence of phase entrainment. Second row, RTs arranged in the same way. Data were smoothed for visualization using a 100-point sliding window (gray lines, SEM resulting from the smoothing). RTs significantly correlated with the delta phase at target onset (circular-linear correlation coefficients and $p$ values are indicated on the plots). Fastest reactions were observed when the delta phase at the target onset fell on the rising slope of delta oscillation, near the negative peak (delta phase in radians at the minimal reaction time, from $10 \%$ to $91 \%:-2.76,-2.68,-2.56,-1.98$, respectively). Third and forth rows, Latency and amplitude of the delta-peak component arranged similarly as in the rows above. These measures also correlated with the delta phase at the target onset, with minimal latency and maximal amplitude near the negative peak of delta oscillation (delta phase at the minimal latency, from $10 \%$ to $91 \%$ : $-2.71,-2.57,-2.14,-2.13$, respectively; delta phase at maximal amplitude, from $10 \%$ to $91 \%$ : $-3.12,-3.11,-2.96,-2.93$, respectively).

supplemental Fig. 1, available at www.jneurosci.org as supplemental material). Cue 1 predicted that the target would either follow after $1350 \mathrm{~ms}$ (early target; $p=0.2$ ) or after $2700 \mathrm{~ms}$ (late target; $p=0.8)$. Cue 2 predicted an early target with $p=0.8$ or a later target with $p=0.2$. Participants $(n=11)$ were instructed to respond to targets as fast as possible. As was expected on the basis of the results of experiment 1 , significantly shorter RTs were obtained for higher- than for lowerprobability early targets (Fig. $1 D$ ).

Experiment 2 allowed us to investigate phase entrainment without confounding stimulus-evoked and motor-related activity by analyzing instantaneous delta-phase values at the time of the expected delivery of early targets on late-target trials (i.e., when no early target was delivered). Delta-band oscillations extracted from the EEG signal recorded at midline electrode locations $(\mathrm{Fz}, \mathrm{Cz}, \mathrm{Pz}$; figures show data from $\mathrm{Cz}$ ) again preceded the expected target onset (Figs. 2N, 3E, F; supplemental Figs. 8-10, available at www.jneurosci.org as supplemental material). Significant phase entrainment was observed in both conditions (Rayleigh's test, $p<0.05$, supplemental Table 2, available at www. jneurosci.org as supplemental material). Similarly to experiment 1 , more concentrated phase distribution was found with $p=0.8$ than with $p=0.2$ early-target probability (permutation test, $p<$ 0.05 ; Fig. $2 I-L$ ). Thus the accuracy of phase locking was stronger at higher target probability.

\section{Task performance correlates with prestimulus delta oscillatory phase}

We showed that the strength of phase entrainment of delta-band oscillations depends on the level at which a stimulus is predicted.
Can this difference in the entrainment of cortical oscillatory activity be directly linked to task performance or is it the consequence of neuronal mechanisms, which are not involved in forming the overt response to the target? To test whether or not the phase entrainment of delta-band oscillations plays a role in target detection, using single trials in experiment 1 , we examined the correlation between the phase of the delta-band oscillation at the onset of the target tone and 1) reaction time as well as 2) latency and 3) peak amplitude of the prominent delta-peak constituent of the P300 ERP response (referred to as the delta-peak; see Fig. $2 M$ ). All three measures significantly correlated with the phase of the prestimulus delta oscillation at each target probability (Fig. 4, see circular-linear correlation coefficients and significance values). Moreover, delta phase-reaction time correlations exhibited only subtle changes and remained highly significant when circular-linear partial correlations were calculated with P300 latency or amplitude as controlling variables (supplemental Tables 9,10 , available at www.jneurosci.org as supplemental material). These results are consistent with a scenario in which prestimulus delta phase exerts an independent influence on both reaction time and P300 response. Figure 4 also shows that fast reaction times coincided with short-latency high-amplitude delta-peaks characterizing best task performance. This combination of behavioral and EEG measures was obtained on trials in which the target was delivered during the rising slope (close to the negative peak) of the delta-band filtered EEG signal (Fig. $2 M$ ). The phase values related to optimal responses (Fig. 4) were close to the mean delta phase values at the expected onset time of the targets in high expectancy conditions (Fig. 2G,H). 
Finally, we tested the laterality of the delta phase effect, as significant lateralization would suggest that motor readiness is a central factor of phase entrainment. We performed the comparison of phase locking of prestimulus delta waves recorded from the left central (C3) and right central (C4) leads in experiment 1. We could not show any significant difference either in the depth of phase modulation (supplemental Fig. 12, available at www. jneurosci.org as supplemental material and supplemental Table 7 , available at www.jneurosci.org as supplemental material) or in the strength of correlation between prestimulus delta phase and (1) reaction time, (2) P300 delta peak latency, (3) P300 delta-peak amplitude (supplemental Table 8, available at www.jneurosci.org as supplemental material). This lack of lateralization indicates that motor readiness may not be the central driving force of the entrainment; however, the entrainment of motor cortical areas to predictable visual stimuli (Saleh et al., 2010) probably does contribute to the entrained delta oscillations analyzed.

\section{Discussion}

The reliability of cue-based conscious expectation improves with increasing predictive value of the cue. Here we report that expectation strengthens delta-band phase-entrainment to and speeds up the detection of relevant stimulus events. Our results further reveal that target events expected with high confidence are likely to 1) coincide with the negative-going phase of the frontocentral delta oscillations, 2) elicit short-latency and high-amplitude delta-peak (P300) ERP responses, and 3) are quickly detected consciously. Therefore, we suggest that the entrainment of cortical delta oscillations to expected stimulus onsets plays an important role in mediating the effects of anticipation on target detection in humans. Our study extends the recent finding that low-frequency oscillations entrain to rhythmic stimuli in macaques (Lakatos et al., 2008) to humans. Moreover, our results go beyond the previous understanding about passive phase-entrainment, providing evidence that phase concentration-and its beneficial effects on event processing - depends on the likelihood of occurrence of the behaviorally significant event, suggesting that phase entrainment is not merely a mechanistic consequence of periodic stimulation.

Our results were obtained by using a rhythmic auditory target detection task. One might argue that this paradigm imposes a temporal pattern on neural activity, however, so do most naturalistic stimuli, including biological motion and vocal communication. Under these predictable circumstances, neuronal oscillations can entrain to the structure of the stimulus stream (Schroeder et al., 2008). It has also been proposed recently that, at least in a set of tasks, where the temporal structure of attended stimuli is rhythmic or predictable, the brain operates in a rhythmic mode, in which low-frequency rhythms provide a general oscillatory framework for stimulus processing (Schroeder and Lakatos, 2009) (see also supplemental Notes, available at www.jneurosci.org as supplemental material). We established the presence of low-frequency oscillations during an auditory target detection task by visualizing delta-band filtered and wide-band single-trial EEG recordings (Fig. 3; supplemental Fig. 13, available at www.jneurosci.org as supplemental material). Because in experiment 1, cue stimuli were always followed by another (cue or target) stimulus in 1200-1500 ms, only a few (usually two, sometimes three) oscillatory cycles could be observed between two stimuli. Thus, one might argue that a higher minimal number of oscillatory cycles is needed to assess the presence of oscillatory activity. Nevertheless, we followed the definitions and terms of previous works related to phase entrainment that implemented similar temporal arrangement of periodic stimuli (Lakatos et al., 2007, 2008, 2009). Moreover, slow oscillations during normal human sleep often comprise only short bouts of low-frequency oscillation, usually consisting of only a few cycles (Lee-Chiong, 2006; Parrino et al., 2006). In many reports, the detection of a single cycle or even a positive or negative half wave justifies the use of the term "oscillation" (Massimini et al., 2004; Mölle et al., 2006). Additionally, that we consider rhythmic low-frequency amplitude fluctuations as oscillations despite the limited number of cycles in experiment 1 is further supported by longer stimulus-free segments in experiment 2, in which interstimulus interval was $2700 \mathrm{~ms}$ in late target trials. In these cases, the continuation of delta-band oscillations after expected, but not delivered target stimuli is clearly visible (Fig. $3 E, F$ ).

Although digital filtering can cause the distortion of the original signals and such effects cannot be completely ruled out in experiment 1 , the design of experiment 2 , in which no stimulusevoked activity could contaminate the prestimulus signals, allowed us to control for such undesired phenomena. Moreover, we performed additional data analysis and simulations to exclude the possibility that filter artifacts substantially affected our results of both experiments, which are detailed in the supplemental material (supplemental Notes, supplemental Figs. 14-16, and supplemental Tables $4-6$, available at www.jneurosci.org as supplemental material).

Delta oscillations $(0.5-3 \mathrm{~Hz})$ are present in the background EEG activity not just in sleep, but in awake state and are thought to be generated by neocortical and thalamocortical networks. These oscillations play an important role in large-scale cortical integration (Bruns and Eckhorn, 2004; Jerbi et al., 2007), especially in the frontocentral parietal anticipatory network (Babiloni et al., 2006; Padilla et al., 2006). Event-related responses are assumed to arise at least partly by superposition of event-related oscillations in several frequency ranges (Karakaş et al., 2000; Schürmann et al., 2001; Gruber et al., 2005; Klimesch et al., 2007). The human P300 ERP component (for review, see Polich, 2007) was observed in a variety of tasks that require stimulus discrimination. In addition to being linked to context update processes (Donchin and Coles, 1988), it was suggested to originate from the neural linkage between stimulus perception and the initiation of the response (Verleger, 1997; Verleger et al., 2005). Although P300 is a compound response consisting of delta, theta and alpha frequency constituents, it is dominated by the delta-band component (Schürmann et al., 2001; Bernat et al., 2007; Ergen et al., 2008; Ishii et al., 2009). Parts of our findings replicate well documented phenomena, including larger P300 response to lower sequence probability events (Mars et al., 2008) (supplemental Figs. 5-7, available at www.jneurosci.org as supplemental material, show that cue events with lower probability of occurrence evoke larger P300 response). However, less frequently occurring cue tones were more probably followed by the target tone, and the more predictable the target was, the larger P300 response it has evoked (Fig. $2 \mathrm{M}$ shows that targets following cue events with lower sequential probability evoke larger delta-band response). The latter effect of different levels of expectancy was also apparent in experiment 2, where the probabilities of the cues within the sequence were set equal.

Our present results suggest that the entrainment of cortical delta oscillations to periodic and expected stimulus onsets plays an important role in mediating the effects of anticipation on target detection in humans when the target onset is predictable, and when events in the attended task occur at time intervals that correspond to the wavelength of delta oscillations $(\sim 1 s)$. Other neural correlates of predictive behavior have been suggested by 
Riehle et al. (1997), reporting that the synchronization of single neurons in the monkey motor cortex is related to predictable stimuli. Although it is not straightforward to make speculative comparisons between human scalp EEG recordings and monkey single-unit data, the synchronization of neuronal ensembles proposed by Riehle et al. could reflect large-scale network synchrony leading to an entrainment of oscillatory activity (see also below). This is in line with recent results indicating large-scale delta phase-coupling between primary motor cortex and multiple brain regions including structures of the frontoparietal circuit and cerebello-thatamo-cortical pathway during the performance of a continuous visuomotor task (Jerbi et al., 2007).

Rhythmic changes in the local field potential reflect the responsiveness of cortical neurons (Lakatos et al., 2005, 2008; Haider et al., 2007). As an example, visual perceptual performance fluctuates along with the phase of background EEG activity (Busch et al., 2009; Mathewson et al., 2009). Coherent oscillations can indicate synchronization of excitable phases within a large-scale network comprising distant neuronal ensembles. Phase synchronization was suggested as a key mechanism of neuronal communication by dynamically linking neurons into functional networks, which process task-relevant information and select behavior during sensory-motor integration (Womelsdorf and Fries, 2006; Womelsdorf et al., 2007). Consequently, the entrainment of the phase of cortical delta oscillations to the predicted time of a stimulus event may facilitate efficacious neuronal communication between the brain areas of sensory processing and response execution. Thus the prestimulus modulation of the delta rhythm may serve as a neural mechanism underlying the faster processing of expected events.

\section{References}

Adrian ED, Matthews BHC (1934) The Berger rhythm: potential changes from the occipital lobes in man. Brain 57:355-384.

Arieli A, Sterkin A, Grinvald A, Aertsen A (1996) Dynamics of ongoing activity: explanation of the large variability in evoked cortical responses. Science 273:1868-1871.

Babiloni C, Brancucci A, Vecchio F, Arendt-Nielsen L, Chen AC, Rossini PM (2006) Anticipation of somatosensory and motor events increases centro-parietal functional coupling: an EEG coherence study. Clin Neurophysiol 117:1000-1008.

Başar E (1980) EEG-brain dynamics. Relation between EEG and brain evoked potentials. Amsterdam: Elsevier.

Bendixen A, Schröger E, Winkler I (2009) I heard that coming: ERP evidence for stimulus driven prediction in the auditory system. J Neurosci 29:8447-8451.

Bernat EM, Malone SM, Williams WJ, Patrick CJ, Iacono WG (2007) Decomposing delta, theta, and alpha time-frequency ERP activity from a visual oddball task using PCA. Int J Psychophysiol 64:62-74.

Bruns A, Eckhorn R (2004) Task-related coupling from high- to lowfrequency signals among visual cortical areas in human subdural recordings. Int J Psychophysiol 51:97-116.

Busch NA, Dubois J, VanRullen R (2009) The phase of ongoing EEG oscillations predicts visual perception. J Neurosci 29:7869-7876.

Buzsáki G (2006) Rhythms of the brain. Oxford: Oxford UP.

Buzsáki G, Draguhn A (2004) Neuronal oscillations in cortical networks. Science 304:1926-1929.

Delorme A, Makeig S (2004) EEGLAB: an open source toolbox for analysis of single-trial EEG dynamics including independent component analysis. J Neurosci Methods 134:9-21.

Donchin E, Coles MG (1988) Is the P300 component a manifestation of context updating? Behav Brain Sci 11:357-374.

Ergen M, Marbach S, Brand A, Baçsar-Eroğlu C, Demiralp T (2008) P3 and delta band responses in visual oddball paradigm in schizophrenia. Neurosci Lett 440:304-308.

Fisher N (1993) Statistical analysis of circular data. Cambridge, UK: Cambridge UP.

Gabor D (1946) Theory of communication. Proc IEE 93:429-457.
Galambos R, Makeig S, Talmachoff PJ (1981) A 40Hz auditory potential recorded from the human scalp. Proc Natl Acad Sci U S A 78:2643-2647.

Gehring WJ, Gratton G, Coles MG, Donchin E (1992) Probability effects on stimulus evaluation and response processes. J Exp Psychol Hum Percept Perform 18:198-216.

Gruber WR, Klimesch W, Sauseng P, Doppelmayr M (2005) Alpha phase synchronization predicts P1 and N1 latency and amplitude size. Cereb Cortex 15:371-377.

Guderian S, Schott BH, Richardson-Klavehn A, Düzel E (2009) Medial temporal theta state before an event predicts episodic encoding success in humans. Proc Natl Acad Sci U S A 106:5365-5370.

Haider B, Duque A, Hasenstaub AR, Yu Y, McCormick DA (2007) Enhancement of visual responsiveness by spontaneous local network activity in vivo. J Neurophysiol 97:4186-4202.

Hasenstaub A, Sachdev RN, McCormick DA (2007) State changes rapidly modulate cortical neuronal responsiveness. J Neurosci 27:9607-9622.

Haslinger R, Ulbert I, Moore CI, Brown EN, Devor A (2006) Analysis of LFP phase predicts sensory response of barrel cortex. J Neurophysiol 96:1658-1663.

Hick WE (1952) On the rate of gain of information. Q J Exp Psychol 4:11-26.

Hurtado JM, Rubchinsky LL, Sigvardt KA (2004) Statistical method for detection of phase-locking episodes in neural oscillations. J Neurophysiol 91:1883-1898.

Hyman R (1953) Stimulus information as a determinant of reaction time. J Exp Psychol 45:188-196.

Ishii R, Canuet L, Herdman A, Gunji A, Iwase M, Takahashi H, Nakahachi T, Hirata M, Robinson SE, Pantev C, Takeda M (2009) Cortical oscillatory power changes during auditory oddball task revealed by spatially filtered magnetoencephalography. Clin Neurophysiol 120:497-504.

Jerbi K, Lachaux JP, N'Diaye K, Pantazis D, Leahy RM, Garnero L, Baillet S (2007) Coherent neural representation of hand speed in humans revealed by MEG imaging. Proc Natl Acad Sci U S A 104:7676-7681.

KarakaşS, Erzengin OU, Başar E (2000) A new strategy involving multiple cognitive paradigms demonstrates that ERP components are determined by the superposition of oscillatory responses. Clin Neurophysiol 111:1719-1732.

Klimesch W, Sauseng P, Hanslmayr S, Gruber W, Freunberger R (2007) Event-related phase reorganization may explain evoked neural dynamics. Neurosci Biobehav Rev 31:1003-1016.

Kruglikov SY, Schiff SJ (2003) Interplay of electroencephalogram phase and auditory-evoked neural activity. J Neurosci 23:10122-10127.

Lakatos P, Shah AS, Knuth KH, Ulbert I, Karmos G, Schroeder CE (2005) An oscillatory hierarchy controlling neuronal excitability and stimulus processing in the auditory cortex. J Neurophysiol 94:1904-1911.

Lakatos P, Chen CM, O'Connell MN, Mills A, Schroeder CE (2007) Neuronal oscillations and multisensory interaction in primary auditory cortex. Neuron 53:279-292.

Lakatos P, Karmos G, Mehta AD, Ulbert I, Schroeder CE (2008) Entrainment of neuronal oscillations as a mechanism of attentional selection. Science 320:110-113.

Lakatos P, O'Connell MN, Barczak A, Mills A, Javitt DC, Schroeder CE (2009) The leading sense: supramodal control of neurophysiological context by attention. Neuron 64:419-430.

Lee-Chiong T (2006) Sleep: a comprehensive handbook. Hoboken, NJ: Wiley.

Mars RB, Debener S, Gladwin TE, Harrison LM, Haggard P, Rothwell JC, Bestmann S (2008) Trial-by-trial fluctuations in the event-related electroencephalogram reflect dynamic changes in the degree of surprise. J Neurosci 28:12539-12545.

Massimini M, Huber R, Ferrarelli F, Hill S, Tononi G (2004) The sleep slow oscillation as a traveling wave. J Neurosci 24:6862-6870.

Mathewson KE, Gratton G, Fabiani M, Beck DM, Ro T (2009) To see or not to see: prestimulus alpha phase predicts visual awareness. J Neurosci 29:2725-2732.

Mölle M, Yeshenko O, Marshall L, Sara SJ, Born J (2006) Hippocampal sharp wave-ripples linked to slow oscillations in rat slow-wave sleep. J Neurophysiol 96:62-70.

Monto S, Palva S, Voipio J, Palva JM (2008) Very slow EEG fluctuations predict the dynamics of stimulus detection and oscillation amplitudes in humans. J Neurosci 28:8268-8272. 
Nobre A, Correa A, Coull J (2007) The hazards of time. Curr Opin Neurobiol 17:465-470.

Ortiz TA, Goodin DS, Aminoff MJ (1993) Neural processing in a threechoice reaction-time task: a study using cerebral evoked-potentials and single-trial analysis in normal humans. J Neurophysiol 69:1499-1512.

Padilla ML, Wood RA, Hale LA, Knight RT (2006) Lapses in a prefrontalextrastriate preparatory attention network predict mistakes. J Cogn Neurosci 18:1477-1487.

Parrino L, Halasz P, Tassinari CA, Terzano MG (2006) CAP, epilepsy and motor events during sleep: the unifying role of arousal. Sleep Med Rev 10:267-285.

Polich J (2007) Updating P300: an integrative theory of P3a and P3b. Clin Neurophysiol 118:2128-2148.

Riehle A, Grün S, Diesmann M, Aertsen A (1997) Spike synchronization and rate modulation differentially involved in motor cortical function. Science 278:1950-1953.

Romei V, Brodbeck V, Michel C, Amedi A, Pascual-Leone A, Thut G (2008) Spontaneous fluctuations in posterior alpha-band EEG activity reflect variability in excitability of human visual areas. Cereb Cortex 18:2010-2018.

Saleh M, Reimer J, Penn R, Ojakangas CL, Hatsopoulos NG (2010) Fast and slow oscillations in human primary motor cortex predict oncoming behaviorally relevant cues. Neuron 65:461-471.

Schroeder CE, Lakatos P (2009) Low-frequency neuronal oscillations as instruments of sensory selection. Trends Neurosci 32:9-18.

Schroeder CE, Lakatos P, Kajikawa Y, Partan S, Puce A (2008) Neuronal oscillations and visual amplification of speech. Trends Cogn Sci 12:106-113.

Schürmann M, Baçsar-Eroglu C, Kolev V, Baçsar E (2001) Delta responses and cognitive processing: single-trial evaluations of human visual P300. Int J Psychophysiol 39:229-239.
Summerfield C, Egner T (2009) Expectation (and attention) in visual cognition. Trends Cogn Sci 13:403-409.

van Dijk H, Schoffelen JM, Oostenveld R, Jensen O (2008) Prestimulus oscillatory activity in the alpha band predicts visual discrimination ability. J Neurosci 28:1816-1823.

van Elswijk G, Kleine BU, Overeem S, Stegeman DF (2007) Expectancy induces dynamic modulation of corticospinal excitability. J Cogn Neurosci 19:121-131.

van Elswijk G, Maij F, Schoffelen JM, Overeem S, Stegeman DF, Fries P (2010) Corticospinal beta-band synchronization entails rhythmic gain modulation. J Neurosci 30:4481-4488.

Vanhatalo S, Palva JM, Holmes MD, Miller JW, Voipio J, Kaila K (2004) Infraslow oscillations modulate excitability and interictal epileptic activity in the human cortex during sleep. Proc Natl Acad Sci U S A 101:5053-5057.

Verleger R (1997) On the utility of P3 latency as an index of mental chronometry. Psychophysiology 34:131-156.

Verleger R, Japkowski P, Wascher E (2005) Evidence for an integrative role of P3b in linking reaction to perception. J Psychophysiol 19:165-181.

Walter WG, Dovey VJ, Shipton H (1946) Analysis of electrical response of human cortex to photic stimulation. Nature 158:340-541.

Will U, Berg E (2007) Brain wave synchronization and entrainment to periodic acoustic stimuli. Neurosci Lett 424:55-60.

Womelsdorf T, Fries P (2006) Neuronal coherence during selective attentional processing and sensory-motor integration. J Physiol Paris 100:182-193.

Womelsdorf T, Schoffelen JM, Oostenveld R, Singer W, Desimone R, Engel AK, Fries P (2007) Modulation of neuronal interactions through neuronal synchronization. Science 316:1609-1612. 